\title{
Variabilidade espacial de atributos físicos em solos de vale aluvial no semiárido de Pernambuco ${ }^{1}$
}

\author{
Karla S. Santos ${ }^{2}$, Abelardo A. A. Montenegro ${ }^{3}$, Brivaldo G. de Almeida ${ }^{4}$, \\ Suzana M. G. L. Montenegro ${ }^{5}$, Tafnes da S. Andrade ${ }^{6}$ \& Robertson V. de P. Fontes Júnior
}

\begin{abstract}
RESUMO
As características físicas e hidráulicas do solo se constituem em condicionantes a planos de manejo agrícola. Objetivou-se avaliar atributos físicos em solos de um vale aluvial e sua variabilidade, no semiárido do Estado de Pernambuco, na bacia hidrográfica do Rio Ipanema. Técnicas estatísticas e geoestatísticas foram utilizadas a fim de se investigar o grau de dependência e a variabilidade espacial dos atributos, em lotes de agricultura familiar. O coeficiente de variação apresentou-se alto para a condutividade hidráulica do solo e médio para a resistência à penetração de raízes e para as frações granulométricas areia, argila e silte. Tal coeficiente foi baixo para a densidade do solo e de partículas e porosidade total. Foram investigadas as estruturas de dependência espacial para as variáveis estudadas, encontrando-se alcances variando de $135 \mathrm{~m}$ para a condutividade hidráulica a $465 \mathrm{~m}$ para o conteúdo de silte. Mapa de isolinhas foi elaborado por krigagem representando a variabilidade espacial da condutividade hidráulica, com moderado grau de dependência espacial. As frações granulométricas areia total e silte (\%) também apresentaram grau de dependência espacial moderado. A resistência à penetração, densidade do solo e de partículas, não apresentaram dependência espacial na escala de investigação adotada. Foi possível identificar regiões de maior aptidão agrícola verificando-se, através do mapeamento, que os solos francos tendem a apresentar elevada resistência à penetração e baixa condutividade hidráulica.
\end{abstract}

Palavras-chave: condutividade hidráulica, resistência do solo à penetração, geoestatística

\section{Spatial variability of physical attributes of soil in alluvial valley of semiarid region of Pernambuco State}

\begin{abstract}
Soil physical and hydraulic characteristics are relevant for agricultural management, particularly at irrigation districts. The objective of this study was to evaluate the spatial variability of physical attributes of soil in an alluvial valley in the semiarid region of Pernambuco State, at the Ipanema River Watershed. Statistical techniques and geostatistics were applied to observe the degree of dependence and the spatial variability of soil physical properties, in plots adopted for communal agriculture. The coefficient of variation for the hydraulic conductivity was high, medium for soil resistance to root penetration and for sand, clay and silt. For bulk density, particle density and porosity, however, such coefficient was low. Spatial dependence structures were investigated, with ranges spanning from $135 \mathrm{~m}$, for soil hydraulic conductivity, to $465 \mathrm{~m}$, for silt content. Contour map was produced by kriging interpolation, representing the spatial variability of hydraulic conductivity, which showed moderate spatial dependence. Resistance to root penetration, soil density and particle distribution presented spatial random behavior, for the adopted spatial scale. It was possible to identify regions with higher agricultural potential. From the produced map, it was highlighted that loam soils usually present higher resistance to root penetration, and low hydraulic conductivity.
\end{abstract}

Key words: hydraulic conductivity, penetration resistance, geoestatistics

\footnotetext{
Parte da dissertação de mestrado do primeiro autor, apresentada à Universidade Federal Rural de Pernambuco, UFRPE

CCAAB/UFRB. Rua Rui Barbosa, 710, Centro, CEP 44380-000, Cruz das Almas, BA. Fone: (75) 3621-3120. E-mail: karlasilvasantos@yahoo.com.br DTR/UFRPE. R. Dom Manoel de Medeiros, s/n, Dois Irmãos, CEP 52171-900, Recife, PE. Fone: (81) 3320-6273. E-mail: abelardo.montenegro@yahoo.com.br Departamento de Agronomia/UFRPE. Fone: (81) 3320-2101. E-mail: brivaldo@depa.ufrpe.br

DEC/UFPE. Av. Acadêmico Hélio Ramos, s/n. Cidade Universitária. CEP 50740-530. Recife, PE. Fone: (81) 2126-7216. E-mail: suzanam@ufpe.br CPRH. Av. Bel. Francisco Pereira Lopes, 235, Brejão, PE, CEP 55325-000. Fone: (87) 3761-0697. E-mail: tafnes.andrade@cprh.pe.gov.br Departamento de Tecnologia Rural/UFRPE. Fone: (81) 3320-6264. E-mail: rr_fontes@hotmail.com
} 


\section{INTRODUÇÃO}

A região semiárida do Nordeste brasileiro apresenta distribuição irregular da precipitação no tempo, altas taxas de evaporação, solos rasos e escassos recursos hídricos; essas características climáticas, pedológicas e hidrológicas limitam o desenvolvimento da agricultura na região.

Os vales aluviais do semiárido apresentam elevado potencial para a pequena agricultura porém são susceptíveis aos processos de acúmulo de sais influenciados pela distribuição espacial de suas características hidráulicas. Em tais áreas a não consideração da variabilidade espacial dos atributos do solo pode afetar a eficiência dos planos de manejo agrícola (Montenegro \& Montenegro, 2006).

Em uma área cultivada existem, além da variabilidade natural, fontes adicionais de heterogeneidade no solo, devido ao manejo adotado (Camargo et al., 2010). Tais variações influenciam o acúmulo de material orgânico, o movimento de água no solo, a compactação e a erosão hídrica (Novaes Filho et al., 2007). Áreas pedologicamente similares podem apresentar variabilidade distinta em seus atributos quando submetidas a diferentes práticas de manejo (Corá et al., 2004).

Investigações enfocando a variabilidade espacial da textura e da condutividade hidráulica em Neossolos foram desenvolvidas por Montenegro \& Montenegro (2006), que abordaram as estruturas de dependência espacial em escala de perímetro irrigado com agricultura familiar, adotado neste estudo. Alcances da ordem de $320 \mathrm{~m}$ foram obtidos para a taxa de infiltração e de $333 \mathrm{~m}$ para classes de solo predominantes, mediante uma malha de amostragem composta de pontos aleatórios e conjuntos de transectos, tendo-se identificado regiões de maior susceptibilidade à salinização. Adicionalmente, Montenegro et al. (2010) abordaram a variabilidade espacial da condutividade elétrica de Neossolo em escala de lote e obtiveram alcances da ordem de dezenas de metros, constatando o melhor desempenho do modelo gaussiano para representar tal dependência espacial.

Em escala de lote em Latossolo do bioma Cerrado, Cavalcante et al. (2011) estudaram a variabilidade espacial da resistência a penetração utilizando malha regular. Verificaram os autores que a resistência à penetração foi influenciada pelo manejo do solo e os modelos teóricos de semivariograma que melhor se ajustaram dependiam da profundidade da amostragem. Para a camada de 0,15 - 0,30 m foram selecionados os modelos esférico e exponencial. Também em Latossolo Guedes Filho et al. (2010), Fidalski et al. (2006) e Silva et al. (2004) caracterizaram a variabilidade espacial da resistência a penetração tal como, também, dos atributos físicos e químicos, adotando malhas regulares com diferentes escalas, tendo observado predominância do modelo esférico para os semivariogramas teóricos.

Cortez et al. (2010) examinaram a dependência espacial da resistência a penetração em Argissolo Amarelo no Vale do Rio São Francisco, ajustando os modelos esférico e exponencial para diferentes profundidades do solo, com alcances variando de 60 a $130 \mathrm{~m}$.

Neste contexto e se considerando a carência de estudos enfocando o comportamento de atributos físicos em Neossolos objetivou-se, com este trabalho, caracterizar a variabilidade espacial de atributos físicos, hidráulicos e de resistência à penetração em solos de um vale aluvial, no semiárido de Pernambuco.

\section{Material e MÉTODOS}

A área de estudo está localizada na Fazenda Nossa Senhora do Rosário, a $230 \mathrm{~km}$ de Recife, PE, com coordenadas geográficas de $8^{\circ} 21^{\prime} 28^{\prime \prime}$ de latitude Sul e $36^{\circ} 41^{\prime} 47^{\prime \prime}$ de longitude Oeste. O clima da região é semiárido quente tipo estepe, BSsh segundo a classificação de Köppen, com precipitação anual média de $730 \mathrm{~mm}$ e evapotranspiração potencial total anual média de $1683 \mathrm{~mm}$.

O aquífero aluvial sobre o qual a área estudada está situada pertence a uma bacia hidrográfica relativamente plana. A espessura média do aquíferoé em torno de $10 \mathrm{~m}$, cerca de $3 \mathrm{~km}$ de extensão e $300 \mathrm{~m}$ de largura. A altitude média da área é $630 \mathrm{~m}$ e a declividade média é de $0,3 \%$.

A agricultura praticada na área é em pequena escala de base familiar, utilizando-se irrigação com águas subterrâneas, principalmente por meio de aspersão convencional observando-se, entretanto, crescente aceitação da microaspersão.

Segundo levantamento pedológico realizado por Ribeiro \& Corrêa (2001), o solo predominante na área é o Neossolo Flúvico. Nas encostas circundantes se encontram solos do tipo Neossolos Litólicos e Regolíticos, e Argissolos, com presença de impedimento (Figura 1). Na região de solos RU6 predomina a classe textural franca com ocorrência de áreas salinas.

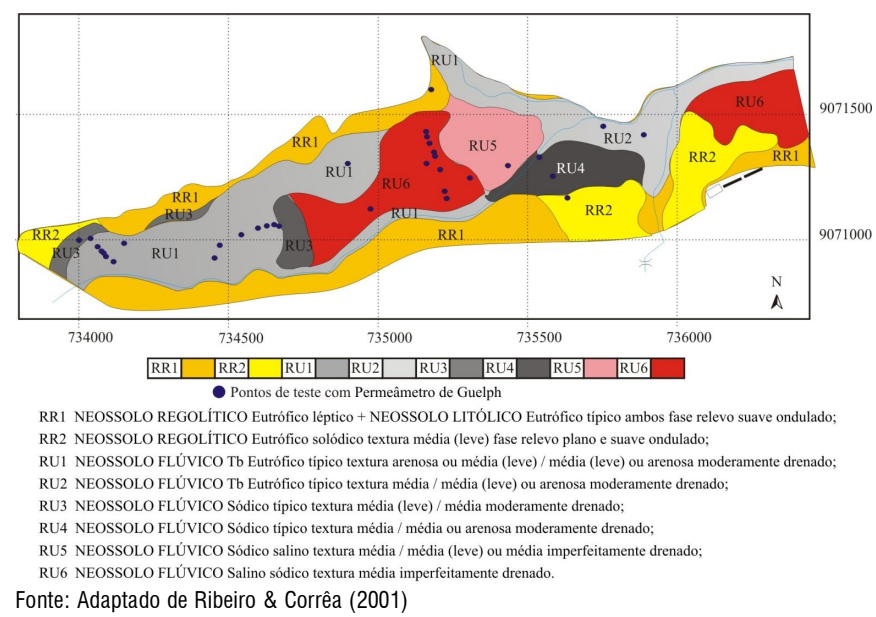

Figura 1. Mapa dos principais solos presentes no vale aluvial e localização dos pontos de monitoramento ao longo dos transectos

A textura dos solos e a baixa declividade dos terrenos no vale limitam as condições de escoamento superficial e favorecem a infiltração nas áreas arenosas.

Os ensaios e coletas foram realizados nos lotes irrigados utilizados por agricultores familiares, que se distribuem transversal e longitudinalmente ao vale, onde é praticada irrigação de forma empírica; esses lotes têm cerca de 1 ha.

A área de estudo possui, instalados, piezômetros distribuídos ao longo de transectos, para fins de monitoramento 
do aquífero subjacente. Optou-se por seguir tais transectos não apenas para a realização dos ensaios de condutividade hidráulica e coleta de amostras de solo mas também para a determinação da resistência do solo à penetração em laboratório, além de análise granulométrica. O espaçamento médio entre os pontos amostrais foi de $64 \mathrm{~m}$ enquanto o espaçamento mínimo foi de $30 \mathrm{~m}$. Estudos anteriores no mesmo vale aluvial, nos quais se utilizou esquema espacial de amostragem semelhante, indicaram o espaçamento de $30 \mathrm{~m}$ como adequado para capturar a estrutura de dependência de propriedades físicas (Montenegro \& Montenegro, 2006). O esquema espacial de transectos foi complementado com pontos aleatórios de modo a aumentar o número de pares de pontos adotados para estimativa das semivariâncias experimentais. Journel \& Huijbregts (1978) recomendam um número mínimo de pares igual a 30, para cada semivariância experimental.

Amostras não deformadas foram coletadas na profundidade de $30 \mathrm{~cm}$ tendo-se utilizado anéis volumétricos de diâmetro e altura de aproximadamente $5 \mathrm{~cm}$, introduzidos ao solo por meio de um amostrador tipo Uhland.

A condutividade hidráulica foi determinada em campo através de um permeâmetro de carga constante tipo Guelph, que opera de acordo com o princípio da garrafa de Marriotte de carga hidráulica constante. Foram realizados os ensaios na profundidade de $30 \mathrm{~cm}$ e com a adoção de quatro cargas hidráulicas: 2,5; 5,0; 7,5 e 10,0 cm, com três leituras subsequentes após sua estabilização, calculando-se a média das três últimas leituras. A condutividade hidráulica do solo saturado $\left(\mathrm{K}_{\mathrm{sat}}\right)$ foi estimada utilizando-se equações propostas para o permeâmetro de Guelph (descritas em Scherpinski et al., 2010).

A determinação da resistência à penetração $(\mathrm{RP})$ foi realizada no Laboratório de Física do Solo da UFRPE na tensão de $6 \mathrm{kPa}$, para a qual os macroporos se encontram vazios, isentos de água; para isto, as amostras contidas nos anéis foram preparadas retirando-se o excesso de solo e, em seguida, colocada uma tela permeável na extremidade inferior dos anéis, de maneira a impedir a perda de solo mas permitindo o fluxo de água/ar. Posteriormente, os anéis foram colocados em uma bandeja para saturação pela elevação gradual da carga hidráulica, pelo tempo de 24 h e só então colocadas na mesa de tensão, visando à determinação da porosidade total (PT) e resistência à penetração (RP).

Sempre que as amostras de solo eram retiradas da mesa de tensão e pesadas, iniciava-se o procedimento de leitura de resistência à penetração por um penetrômetro eletrônico de bancada, composto de um atuador linear elétrico com motor de passo, um painel para controle da velocidade, uma base metálica para sustentação do conjunto mecânico e da amostra durante o ensaio além de uma célula de carga com capacidade nominal de $20 \mathrm{~kg}$, acoplada a um microcomputador. Adotou-se haste de 4,02 mm de diâmetro. A velocidade de penetração foi de $10 \mathrm{~mm} \mathrm{~s}^{-1}$ medindo-a da superfície até a profundidade de 4,5 $\mathrm{cm}$ e os valores iniciais foram descartados por conta do efeito de bordadura. Aproveitaram-se os dados dos $3 \mathrm{~cm}$ centrais da amostra calculando-se a média da resistência do solo à penetração das várias medições realizadas neste intervalo.

Após leituras das RP as amostras foram levadas à estufa, na qual foram secadas a uma temperatura de $105{ }^{\circ} \mathrm{C}$ até a estabilização, para determinação do conteúdo da massa de solo seco e assim se obter a densidade do solo (Ds) conforme EMBRAPA(1997).

A PT, percentual da amostra de solo, foi definida a partir dos dados e da densidade de partículas (Dp).

Verificaram-se a estatística descritiva clássica dos dados e a aderência à distribuição Normal, segundo o teste de Kolmogorov-Smirnov, constatando-se que a variabilidade dos atributos foi classificada por meio dos valores do coeficiente de variação, segundo Warrick \& Nielsen (1980), como baixa (CV $<12 \%)$, média $(12 \leq \mathrm{CV} \leq 62 \%)$ e alta $(\mathrm{CV}>62 \%)$. Foram construídos, também, box-plots para as variáveis condutividade hidráulica e resistência à penetração, de modo a analisar a ocorrência de valores discrepantes e os valores dos quartis da distribuição.

Após a verificação de possíveis tendências espaciais nos dados, as semivariâncias experimentais foram estimadas utilizando-se a ferramenta GEO-EAS (Englund \& Sparks, 1991) e GS+ (Robertson, 1998), segundo a Eq. 1, conforme Journel \& Huijbregts (1978):

$$
\gamma(h)=\frac{1}{2 N(h)} \sum_{i=1}^{N(h)}[Z(x+h)-Z(x)]^{2}
$$

em que:

$\mathrm{N}(\mathrm{h})$ - número de pares

$\mathrm{Z}(\mathrm{x}+\mathrm{h})$ e $\mathrm{Z}(\mathrm{x})$ - valores da propriedade de interesse nas locações $(x+h)$ e $(x)$

Em todos os casos, adotou-se $\mathrm{N}(\mathrm{h})>30$, o que tornou necessário, para alguns atributos, a junção de classes consecutivas de distâncias.

Testaram-se, após a obtenção dos semivariogramas experimentais, os modelos gaussiano, esférico e exponencial. Para a seleção dos modelos foram utilizados: o ajuste, através do método das tentativas (subjetivo), o modelo GEO-EAS e a técnica dos mínimos quadrados ponderados (Eq. 2) proposta por Cressie (1985):

$$
f=\sum_{j=1}^{K} \frac{N\left(h_{j}\right)}{\left(\gamma^{*}\left(h_{j}\right)\right)^{2}} \times\left(\gamma^{*}\left(h_{j}\right)-\gamma\left(h_{j}\right)\right)^{2}
$$

em que:

$\mathrm{N}\left(\mathrm{h}_{\mathrm{j}}\right)$ - número de pares da distância de separação hj

K - número de classes de distância

$\gamma^{*}\left(\mathrm{~h}_{\mathrm{j}}\right)$ - semivariância teórica

$\gamma\left(h_{\mathrm{j}}\right)$ - semivariância experimental

Para adoção da Eq. 2, foi utilizado o software Statistica 6 (StatSoft, 2001); em seguida, procedeu-se à validação dos semivariogramas teóricos ajustados, através da ferramenta GEO-EAS (Englund \& Sparks, 1991), verificando-se se os erros padronizados apresentaram média próxima a zero e desviopadrão próximo à unidade, segundo o critério de Jack-Knifing (Vauclin et al., 1983). 
Analisaram-se a estrutura e a dependência espacial por meio de semivariogramas ajustados, em que a dependência espacial é definida pelo alcance $\left(\mathrm{A}_{0}\right)$, pelo efeito pepita $\left(\mathrm{C}_{0}\right)$ e pelo $\operatorname{patamar}\left(\mathrm{C}+\mathrm{C}_{0}\right)$.

No tocante ao grau de dependência espacial (GD), utilizouse a classificação proposta por Cambardella et al. (1994), na qual determinado atributo pode ser considerado exibindo forte, moderada ou fraca dependência espacial, em função da relação entre o efeito pepita puro e o patamar do semivariograma ajustado. A análise da relação $\mathrm{C}_{0} /\left(\mathrm{C}_{0}+\mathrm{C}_{1}\right)$ permite quantificar a proporção do componente aleatório $\left(\mathrm{C}_{0}\right)$ na variância total $\left(\mathrm{C}_{0}+\mathrm{C}_{1}\right)$.

A dependência espacial pode ser classificada em forte, se $\mathrm{GD} \leq 25 \%$; moderada, se $25 \%<\mathrm{GD} \leq 75 \%$ e fraca, se GD $>75 \%$.

Os atributos foram mapeados por krigagem através do software Surfer (Golden Software, 2002).

Realizou-se, com base nos modelos teóricos validados, uma análise exploratória dos atributos considerando-se círculos de interpolação (subdivididos em octantes) de raio máximo correspondente ao valor obtido para o alcance dos semivariogramas.

\section{RESULTADOS E DisCUSSÃO}

Os resultados da estatística descritiva se acham na Tabela 1; os atributos apresentaram distribuição Normal, com exceção da condutividade hidráulica, que se comportou segundo uma distribuição LogNormal.

Elevados valores de condutividade hidráulica encontrados foram muito superiores ao valor do terceiro quartil da distribuição porém não devem ser filtrados como valores discrepantes. Com efeito verificam-se, em campo, contrastes marcantes de textura e de propriedades hidráulicas, que são comuns em planícies de inundação fluvial. No tocante à distribuição de valores de resistência à penetração, não foram detectados valores superiores a 1,4 MPa. Valores de resistência do solo à penetração das raízes de 2,0 a 4,0 MPa, que segundo Tavares Filho \& Tessier (2010), podem restringir ou mesmo impedir o crescimento e o desenvolvimento das raízes.

Adotando-se o critério de classificação proposto por Warrick \& Nielsen (1980) para o coeficiente de variação (CV), verificou-se que a condutividade hidráulica apresentou alta variabilidade, com valor de CV de $261 \%$, evidenciando uma alta heterogeneidade para os solos da área de estudo. Pode-se observar, na Tabela 1, que a razão média/mediana de $\mathrm{K}_{\text {sat }}$ está acima de 1.

Jury et al. (1991) verificaram que o coeficiente de variação para a condutividade hidráulica em solo saturado variou de 48 a $320 \%$. Abreu et al. (2004) encontraram o valor de 62,7\% para o coeficiente de variação da condutividade hidráulica no solo saturado, determinada em campo com a utilização do permeâmetro de Guelph, na profundidade de $0,15 \mathrm{~m}$. A condutividade hidráulica do solo saturado depende do espaço poroso e este parâmetro varia bastante de solo para solo e, também, para o mesmo solo, em função das variações estruturais. O elevado grau de variabilidade da condutividade hidráulica tem sido atestado na literatura, observando-se comportamento Log-Normal desta variável (Woodbury \& Sudicky, 1991), a qual depende da escala adotada em sua estimativa. No caso de estimativas pontuais, como aquelas obtidas a partir do permeâmetro de Guelph, é consistente encontrar-se elevada variabilidade principalmente em se tratando de uma planície aluvial, formada a partir de sucessivas deposições de material de textura variável.

Com relação à variável $\mathrm{Dp}$, com coeficiente de variação igual a $1,3 \%$, foi a que apresentou menor variabilidade dentre as estudadas. Os baixos valores para o CV demonstram menor heterogeneidade desse atributo para a área de estudo. De maneira geral, as variáveis Ds, Dp e Pt apresentam coeficiente de variação relativamente baixo, segundo o critério de classificação para o coeficiente de variação proposto por Warrick \& Nielsen (1980).

As variáveis areia, silte e argila apresentaram média variabilidade, resultados que concordam com os relatados por Vieira et al. (2007), para esses atributos, em estudo realizado em uma microbacia hidrográfica, com Cambissolo Háplico, em sua maior parte, Argissolo Vermelho-Amarelo Distrófico típico e Gleissolo Háplico.

Concorrem para esta média variabilidade o uso diversificado do solo com adoção de implementos agrícolas, e os processos geomorfológicos de transporte e sedimentação, que propiciam uma homogeneização maior das percentagens de areia e argila.

Tabela 1. Parâmetros da estatística descritiva dos atributos físicos do solo

\begin{tabular}{|c|c|c|c|c|c|c|c|c|c|}
\hline \multirow{2}{*}{ Parâmetros estatísticos } & Areia & Argila & Silte & Ds & Dp & \multirow{2}{*}{$\begin{array}{l}\text { PT } \\
\%\end{array}$} & \multirow{2}{*}{$\begin{array}{c}\mathrm{RP} \\
\mathrm{MPa}\end{array}$} & $\mathrm{K}_{\text {sat }}$ & $\log \left(K_{\text {sat }}\right)$ \\
\hline & & $\%$ & & \multicolumn{2}{|c|}{$\mathrm{g} \mathrm{cm}^{-3}$} & & & \multicolumn{2}{|c|}{$\mathrm{cm} \mathrm{h}^{-1}$} \\
\hline Média & 58,04 & 19,83 & 22,10 & 1,54 & 2,66 & 41,98 & 0,65 & 12,14 & 0,41 \\
\hline Mediana & 58,82 & 19,59 & 21,40 & 1,56 & 2,66 & 41,78 & 0,57 & 3,65 & 0,56 \\
\hline Moda & - & 17,66 & - & - & - & - & 1,21 & - & - \\
\hline Desvio Padrão & 14,50 & 3,30 & 11,50 & 0,08 & 0,03 & 2,95 & 0,33 & 31,69 & 0,80 \\
\hline CV $(\%)$ & 24,98 & 16,67 & 51,90 & 5,30 & 1,30 & 7,02 & 51,68 & 261,00 & 195,14 \\
\hline Assimetria & $-0,58$ & 0,46 & 0,55 & 0,20 & 0,07 & $-0,20$ & 0,41 & 4,94 & 0,03 \\
\hline Curtose & $-0,15$ & 0,12 & $-0,26$ & 0,43 & $-1,17$ & 0,42 & $-0,82$ & 26,32 & $-0,36$ \\
\hline 1. ${ }^{\circ}$ Quartil & 48,61 & 17,64 & 13,10 & 1,49 & 2,63 & 40,18 & 0,39 & 0,66 & $-0,17$ \\
\hline 3. ${ }^{\circ}$ Quartil & 69,56 & 21,52 & 29,80 & 1,58 & 2,69 & 43,90 & 0,91 & 7,62 & 0,88 \\
\hline Máximo & 83,79 & 27,93 & 49,20 & 1,74 & 2,72 & 48,49 & 1,32 & 179,88 & 2,25 \\
\hline Mínimo & 22,87 & 13,81 & 2,38 & 1,36 & 2,61 & 34,94 & 0,09 & 0,06 & $-1,21$ \\
\hline $\mathrm{N}^{0}$ amostras & 36 & 36 & 36 & 36 & 36 & 36 & 36 & 33 & 33 \\
\hline Teste W & 0,96 & 0,96 & 0,96 & 0,96 & 0,95 & 0,98 & 0,94 & 0,37 & 0,98 \\
\hline$P<W$ & 0,26 & 0,30 & 0,27 & 0,38 & 0,11 & 0,81 & 0,21 & 0,00 & 0,97 \\
\hline
\end{tabular}

$\mathrm{K}_{\text {sat }}$ - condutividade hidráulica saturada; Ds - densidade do solo; PT - porosidade total; RP - resistência à penetração; Dp - densidade das partículas 
O silte apresentou CV maior que as frações areia e argila; ressalta-se que esta maior variabilidade para o silte pode estar relacionada com sua maior mobilidade no solo, e deposição na planície aluvial.

A variável resistência do solo à penetração das raízes (RP) apresentou 51,68\% para o valor do CV (Tabela 1), considerada de média variabilidade.

Este resultado concorda com o de Silva et al. (2004), que encontraram valores de CV de 44\%, para a RP em Argissolo Vermelho Amarelo e Latossolo Vermelho. Guedes Filho et al. (2010) encontraram valores de CV de $44,92 \%$ para a RP em Latossolo Vermelho, avaliada na profundidade de $0-20 \mathrm{~cm}$, valor este similar ao demonstrado neste estudo.

A Figura 2 apresenta os box plots para as variáveis $\log \mathrm{K}_{\mathrm{sat}}$ e RP, possibilitando visualização da distribuição dos quartis para essas variáveis.

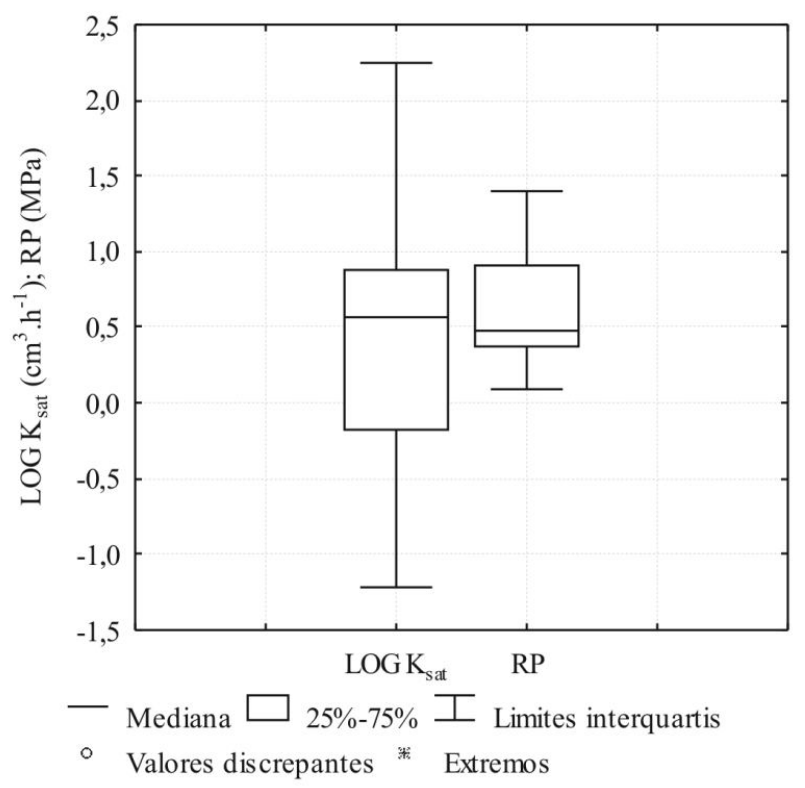

Figura 2. Box plots do Log Ksat e de resistência a penetração (RP) para o vale aluvial

Os semivariogramas experimentais e os semivariogramas teóricos ajustados para os atributos em discussão, estão apresentados nas Figuras 3 e 4, para a textura, resistência à penetração e condutividade hidráulica.

Para as frações areia e silte verificou-se que os modelos gaussianos e esféricos conduziram a alcances excessivamente elevados, superiores às dimensões do domínio, razão pela qual optou-se por considerar modelos exponenciais, com base, inclusive, nos resultados de Montenegro \& Montenegro (2006), que ajustaram e validaram modelos exponenciais para classes de textura, na mesma área aluvial. Apenas o método de ajuste a sentimento produziu modelo exponencial com adequado alcance, tanto para areia quanto para silte, conforme apresentado na Figura 3. Salienta-se que, para os ajustes segundo o critério dos mínimos quadrados ponderados, a presença de maior número de pares para distâncias de separação elevadas influenciou na obtenção de alcances excessivos para as texturas. Em todos os casos o número de pares para cada distância média de separação adotada foi suficiente para a

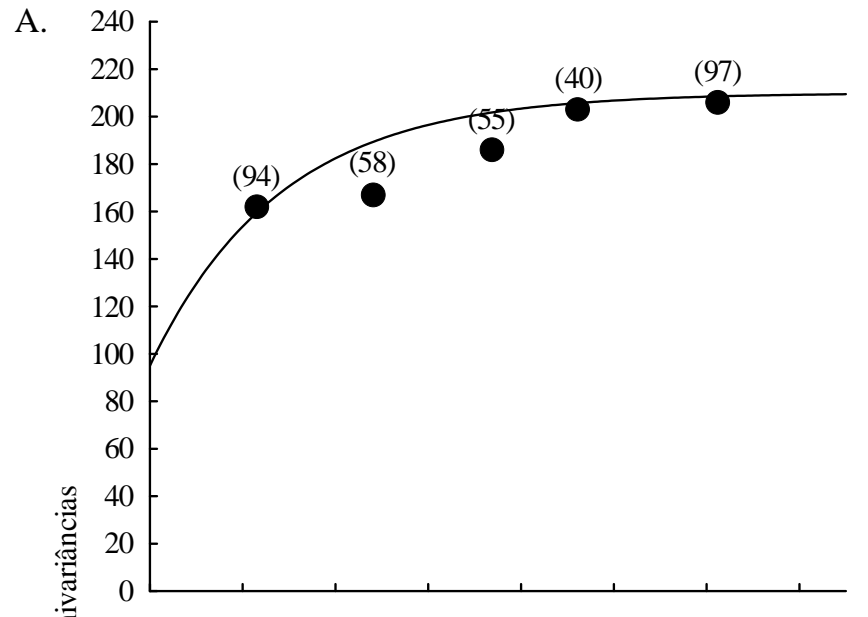

B.

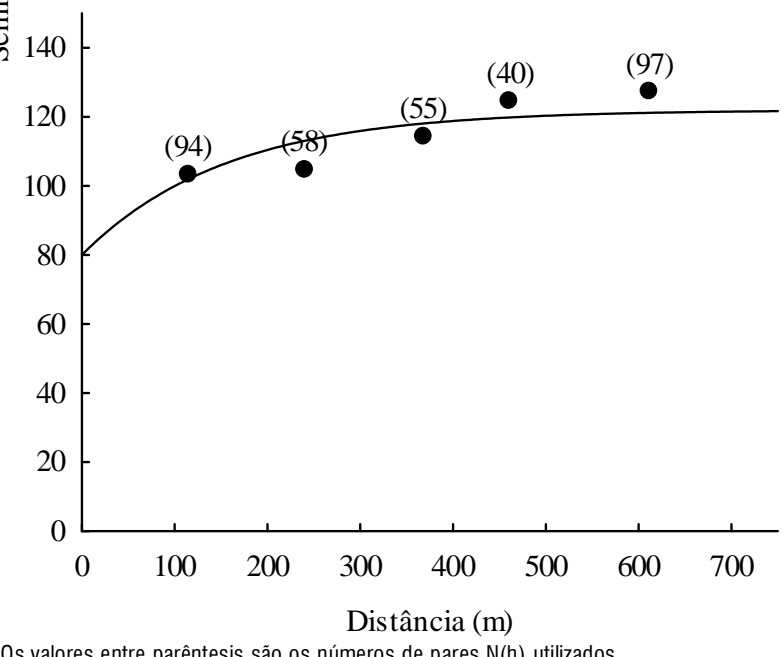

Obs.: Os valores entre parêntesis são os números de pares N(h) utilizados

Figura 3. Semivariogramas exponenciais ajustados a sentimento, para areia (A) e silte (B)

estimativa da semivariância experimental, conforme Journel \& Huijbregts (1978); apesar disto, o número relativamente reduzido de amostras requereu a combinação de classes consecutivas de distâncias, o que limitou o detalhamento das semivariâncias experimentais próximo à origem. Para estudos posteriores recomenda-se não apenas o aumento do número de amostras mas também a redução do espaçamento mínimo entre elas.

Preferiu-se selecionar o modelo gaussiano para a condutividade hidráulica, ajustado segundo a técnica dos mínimos quadrados ponderados com base não apenas no elevado valor do coeficiente de determinação como também nos resultados anteriormente obtidos por Montenegro \& Montenegro (2006) no mesmo vale, a partir de testes de infiltração com duplo cilindro infiltrômetro. Igualmente foi pré-escolhido o modelo gaussiano para a RP obtido a partir do método dos mínimos quadrados, que exibiu elevado $\mathrm{R}^{2}$ (Figura 4).

Já os demais atributos Dp, Ds e PT, analisados variograficamente, apresentaram efeito pepita puro. Assim, pode-se assumir que há independência entre amostras; no entanto não significa, necessariamente, que não haja estrutura de variância, podendo ocorrer dependência espacial em uma distância menor que a adotada entre os pontos de amostragem (Vieira, 2000).

Os semivariogramas teóricos pré-selecionados foram submetidos à validação através da técnica de Jack-knifing 


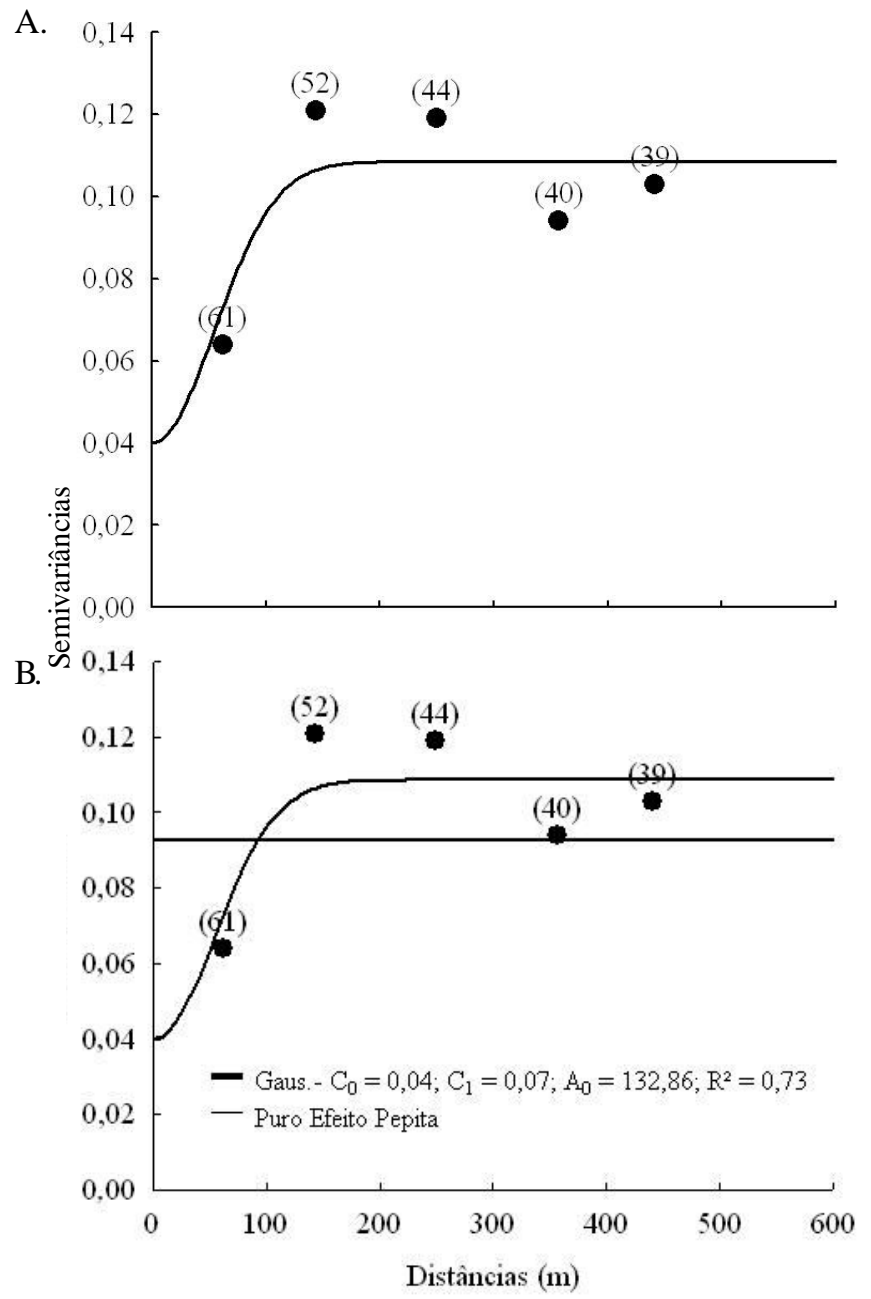

Os valores entre parêntesis são os números de pares N(h) utilizados

Figura 4. Semivariogramas ajustados através dos mínimos quadrados ponderados para log (Ksat) (A) e resistência à penetração (B)

(Vauclin et al., 1983). Os valores da média e do desvio-padrão normalizados estão fornecidos na Tabela 2 e são suficientemente próximos de zero e um, respectivamente (condição de não-tendenciosidade) para as texturas e $\log \left(\mathrm{K}_{\mathrm{sat}}\right)$; entretanto, não ocorreu uma validação adequada para a resistência à

Tabela 2. Resultados da validação cruzada para o Logaritmo da $\mathrm{K}_{\mathrm{Sat}^{\prime}}$ areia, silte e RP (Teste de Jack-Knifing)

\begin{tabular}{lcc}
\hline \multirow{2}{*}{ Atributos } & \multicolumn{2}{c}{ XVALID } \\
\cline { 2 - 3 } & Média dos resíduos & Desvio padrão dos resíduos \\
Log $\mathrm{K}_{\text {sat }}$ & $-0,03$ & 1,01 \\
Areia & 0,09 & 0,92 \\
Silte & $-0,02$ & 0,99 \\
RP & $-0,20$ & 0,47 \\
\hline
\end{tabular}

Log $\mathrm{K}_{\text {Sat }}$ - logaritmo da condutividade hidráulica do solo saturado; XValid - validação cruzada; $\mathrm{RP}$ - resistência a penetração penetração, devendo-se considerar, então, esta variável, como exibindo efeito pepita puro com base na amostragem realizada. A Tabela 3 apresenta os parâmetros dos semivariogramas selecionados e validados, para este estudo.

Embora para algumas variáveis estudadas, as semivariâncias experimentais correspondendo à menor distância de separação sejam relativamente elevadas, os semivariogramas teóricos selecionados para a textura e $\log \left(\mathrm{K}_{\text {sat }}\right)$ foram devidamente validados podendo-se considerá-los adequados para representar as estruturas espaciais em questão.

O alcance da dependência espacial é um parâmetro importante no semivariograma uma vez que indica a zona de influência de uma amostra, ou seja, define a distância máxima até onde o valor de uma variável possui relação de dependência espacial com seus vizinhos e varia aproximadamente de 135 a $465 \mathrm{~m}$, para $\log \left(\mathrm{K}_{\mathrm{sat}}\right)$ e silte, mostrando a extensão da estrutura de dependência espacial das variáveis.

Montenegro \& Montenegro (2006) realizaram pesquisa na mesma área e encontraram alcance de $320 \mathrm{~m}$ para a velocidade de infiltração básica, ajustando os dados ao modelo gaussiano, como já comentado. O maior valor de alcance é justificável considerando-se as diferentes escalas de suporte adotadas nas avaliações da condutividade hidráulica quando se comparam avaliações com permeâmetro de Guelph (da ordem de centímetros) e com infiltrômetro de duplo cilindro (da ordem de dezenas centímetros). Scherpinski et al. (2010) também constataram diferenças entre os alcances da condutividade hidráulica (96,22 m) e da infiltração de água no solo (206,32 m), utilizando um permeâmetro de Guelph em um Latossolo Vermelho.

Para as classes de solo, Montenegro \& Montenegro (2006) encontraram escala de dependência superior a $300 \mathrm{~m}$ analisando a variabilidade espacial das classes de solo franco e francoarenoso, no mesmo vale aluvial.

As características variacionais encontradas para RP estão associadas à sua alta variabilidade na área, em escalas não detectadas pela amostragem. Como já comentado, Cortez et al. (2010) encontraram alcances a partir de $60 \mathrm{~m}$ enquanto Grego \& Vieira (2005) ajustaram os semivariogramas de RP ao modelo esférico, com alcance de 8,75 m, em um Latossolo Vermelho; já neste trabalho a variabilidade foi aleatória e não apenas a classe textural interfere em tal variabilidade mas também as condições de cultivo agrícola, apresentando elevada heterogeneidade no perímetro.

Wojciechowski et al. (2009) observaram que a densidade do solo não indicou autocorrelação entre os pontos amostrados revelando um efeito pepita puro, em uma área de floresta com presença de Neossolo Litólico.

A Tabela 4 apresenta a matriz de correlação para os atributos podendo-se notar baixa correlação global entre os mesmos.

Tabela 3. Parâmetros da análise semivariográfica dos atributos físicos do solo

\begin{tabular}{llcccccc}
\hline Atributos & Modelo & $\mathbf{C}_{\mathbf{0}}$ & $\mathbf{C}$ & $\mathbf{A}_{\mathbf{0}} \mathbf{( m )}$ & $\mathbf{R}^{\mathbf{2}}$ & $\mathbf{G D}$ (\%) & Classificação \\
Log Kat & Gaussiano & 0,31 & 0,38 & 135,03 & 0,73 & 44,9 & Moderada \\
Areia total & Exponencial & 95,00 & 115,00 & 420,00 & 0,76 & 45,2 & Moderada \\
Silte & Exponencial & 80,00 & 42,00 & 465,00 & 0,72 & 65,6 & Moderada \\
\hline
\end{tabular}

Log $\left(K_{\text {sat }}\right)$ - logaritmo da condutividade hidráulica saturada; $G D$ - grau de dependência; $C_{0}$ - efeito pepita; $C$ - patamar; $A_{0}$ - alcance 
Tabela 4. Matriz de correlação entre principais atributos estudados

\begin{tabular}{lrrrrr}
\hline & RP & Areia & Silte & Argila & $\log \left(\mathbf{K}_{\text {sat }}\right)$ \\
RP & 1,00 & & & & \\
Areia & 0,07 & 1,00 & & & \\
Silte & $-0,09$ & $-0,99$ & 1,00 & & \\
Argila & $-0,01$ & $-0,93$ & 0,88 & 1,00 & \\
log $\mathrm{K}_{\text {Sat }}$ & 0,38 & 0,20 & $-0,21$ & $-0,13$ & 1,00 \\
\hline
\end{tabular}

$\mathrm{K}_{\mathrm{sat}}$ - condutividade hidráulica saturada; Ds - densidade do solo; PT - porosidade total; RP - resistência à penetração; Dp - densidade das partículas

Visando observar a distribuição espacial da $\mathrm{K}_{\text {sat }}$ e RP, confeccionou-se um mapa exploratório adotando-se a técnica da krigagem, para $\log \left(\mathrm{K}_{\text {sat }}\right)$ (Figura 5), e com raio de interpolação limitado ao alcance do semivariograma. A malha adotada e as estruturas variacionais obtidas possibilitaram mapear os atributos nas regiões intensivamente cultivadas no vale aluvial, particularmente nas unidades pedológicas RU1 e RU6; entretanto, é importante frisar que as extrapolações aqui obtidas são de caráter exploratório servindo de indicativo preliminar para subsidiar a identificação de regiões potencialmente críticas a serem investigadas definindo-se, assim, locais para futuras coletas.

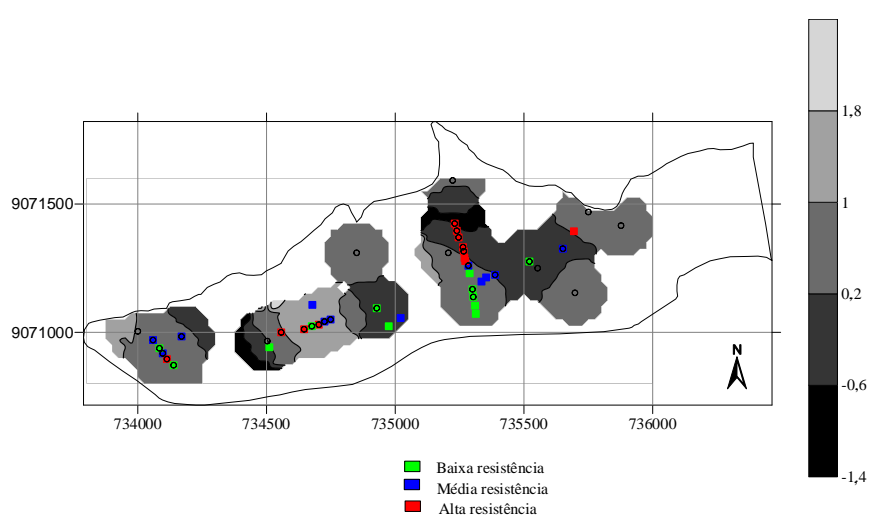

Figura 5. Mapa exploratório de Log $\left(\mathrm{K}_{\mathrm{sat}}\right)$, com destaque para as avaliações de resistências à penetração. Baixa resistência: $\mathrm{RP}<0,42 \mathrm{MPa}$; Média resistência: 0,42 $\leq \mathrm{RP}$ $<0,77 \mathrm{MPa}$ e Alta resistência: $0,77 \leq \mathrm{RP} \leq 1,40 \mathrm{MPa}$

As áreas mais críticas para manejo entre as coordenadas longitudinais 735000 e 735500 , apontadas por Montenegro \& Montenegro (2006), exibem reduzida condutividade hidráulica. Verifica-se, para a RP, seu aumento, à medida em que se aproxima do limite Norte do vale, particularmente na unidade pedológica RU6, que é de solos salinos sódicos. Os solos dominantes nesta região são de classe textural franca.

Em termos de unidades pedológicas se apresentam, na Figura 6, os box-plots por unidades de solo para a RP. Verifica-se que os box-plots da resistência à penetração nas duas classes pedológicas, embora com primeiro e segundo quartis semelhantes, apresentam distinção no terceiro quartil e na dispersão de valores.

Embora se verifiquem diferenças nas distribuições estatísticas da resistência à penetração por unidade pedológica enfatizandose as unidades RU1 e RU6 constata-se, pelo teste de Tukey a $5 \%$, que não ocorre diferença significativa entre as médias de RP nessas duas unidades.

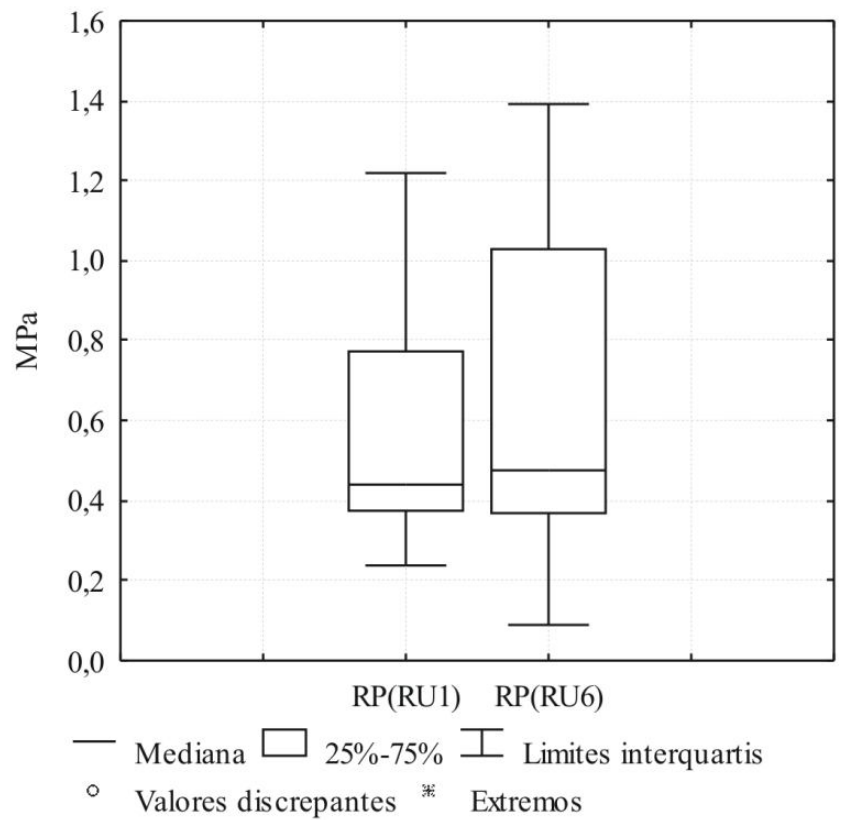

Figura 6. Box-plots da resistência à penetração (RP) por classe pedológica, no vale aluvial

\section{CONCLusões}

1. Os lotes do domínio aluvial apresentando maiores valores de resistência à penetração e menores condutividades hidráulicas, se distribuem próximo ao limite Norte do vale, em área de solo salino sódico e de classe textural franca.

2. Dentre os atributos analisados, a condutividade hidráulica foi o que apresentou maior coeficiente de variação.

3. No estudo da variabilidade espacial dos atributos físicos do solo as variáveis que apresentaram dependência espacial foram o logaritmo da condutividade hidráulica e as frações granulométricas areia e argila, não se detectando, na escala e amostragem adotadas, dependência para resistência à penetração, densidade do solo e porosidade total, que variaram de forma aleatória.

4. A amostragem adotada possibilitou a investigação exploratória das regiões mais críticas, em que estudos complementares devem ser realizados.

\section{Agradecimentos}

Os autores agradecem o apoio do Conselho Nacional de Desenvolvimento Científico e Tecnológico - CNPq/CT-HIDRO, da CAPES, da Finep e da Universidade Federal Rural de Pernambuco - UFRPE, para a realização desta pesquisa.

\section{LITERATURA CITADA}

Abreu, S. L.; Reichert, J. M.; Reinert, D. J. Escarificaçãomecânica e biológica para a redução da compactação em Argissolo franco arenoso sob plantio direto. Revista Brasileira de Ciência doSolo, v.28, p.519-531, 2004. 
Camargo, L. A.; Marques Júnior, J.; Pereira, G. T. Spatial variability of physical attributes of an Alfisol under different hillslope curvatures. Revista Brasileira de Ciência do Solo, v.34, p.617-630, 2010.

Cambardella, C. A.; Moorman, T. B.; Novak, J. M.; Parkin, T. B.; Karlen, D. L.; Turco, R. F.; Konopka, A. E. Field scale variability of soils properties in central Iowa soils. Soil Science Society American Journal, v.58, p.1501-1511, 1994.

Cavalcante, E. G. S.; Alves, M. C.; Souza, Z. M.; Pereira, G. Variabilidade espacial de atributos físicos do solo sob diferentes usos e manejos. Revista Brasileira de Engenharia Agrícola e Ambiental, v.15, p.237-243, 2011.

Corá, J. E.; Araújo, A. V.; Pereira, G. T.; Beraldo, J. M. G. Variabilidade espacial de atributos do solo para adoção do sistema de agricultura de precisão na cultura de cana-deaçúcar. Revista Brasileira de Ciência do Solo, v.28, p.10131021, 2004

Cortez, J. W.; Ferreira, B. J. M.; Olszevski, N.; Lopes, H. L.; Nagahama, H. J. Variabilidade espacial da resistência mecânica à penetração em Argissolo Amarelo no semiárido nordestino. Nucleus, v.7, p.145-154, 2010.

Cressie, N. D. Fitting models by weighted least squares. Journal of Mathematical Geology, v.17, p.563-586, 1985.

EMBRAPA - Empresa Brasileira de Pesquisa Agropecuária. Manual de métodos de análises de solo. 2.ed. Rio de Janeiro: Centro Nacional de Pesquisa de Solos, 1997. 212p.

Englund, E. J.; Sparks, A. Geo-EAS (Geostatistical Environmental Assessment Software). Users's guide. Las Vegas: U.S. Environmental Monitoring Systems Laboratory, 1991. EPA/600/4-88/033a.

Fidalski, J.; Tormena, C. A.; Gonçalves, C. A.; Oliveira Júnior, R. S. Variabilidade da resistência à penetração e da taxa de estratificação de carbono orgânico do solo em um Latossolo Vermelho eutroférrico. Ciência Rural, v.36, p.1773-1779, 2006.

Grego, C. R.; Vieira, S. R. Variabilidade espacial de propriedades físicas do solo em uma parcela experimental. Revista Brasileira de Ciência do Solo, v.29, p.169-177, 2005.

Guedes Filho, O.; Vieira, S. R.; Chiba, M. K.; Nagumo, C. H.; Dechen, C. F. Spatial and temporal variability of crop yield and some rhodic hapludox properties under no-tillage. Revista Brasileira de Ciência do Solo, v.34, p.1-14, 2010.

Golden Software - Surfer 8.0: Contouring and 3nd surface mapping for scientists and engineers. User's Guide. New York: 2002.619p.

Journel, A. G.; Huijbregts, C. J. Mining Geostatistics. Londres: Academic Press, 1978. 600p.

Jury, W.A.; Gardner, W.R., Gardner, W.H. Soil physics. 5 ed. Ney York: John Wiley; Sons, 1991. 328p.

Montenegro, A. A. A.; Netto, M. L. C.; Montenegro, S. M. G.; Silva, E. F. F.; Fontes Júnior, R. V. P. Avaliação da salinidade de Neossolo usando-se dispositivo de indução eletromagnética. Revista Brasileira de Engenharia Agrícola eAmbiental, v.14, p.608-617, 2010.
Montenegro, S. M. G. L.; Montenegro, A. A. A. Variabilidade espacial de classes de textura, salinidade e condutividade hidráulica de solos em planície aluvial. Revista Brasileira de Engenharia Agrícola eAmbiental, v.10, p.30-37, 2006.

Novaes Filho, J. P.; Couto, E. G.; Oliveira, V. A.; Johnson, M. S.; Lehmann, J.; Riha, S. S. Variabilidade espacial de atributos físicos de solo usada na identificação de classes pedológicas de microbacias na Amazônia meridional. Revista Brasileira de Ciência do Solo, v.31, p.91-100, 2007.

Ribeiro, M. R.; Corrêa, M. M. Levantamento pedológico ultra detalhado do vale aluvial da Fazenda Nossa Senhora do Rosário. In: Montenegro, A., Relatório Final de Pesquisa do PNEPG/ CNPq, Recife: Universidade Federal Rural de Pernambuco. 2001.35p.

Robertson, G. P. GS+ geostatistics for the environmental sciences: GS+ user's guide. Plainwell: Gamma Design Software, 1998. 152p.

Scherpinski, C; Uribe-Opazo, M.A.; Vilas Boas, M.A.; Sampaio, S.C.; Johann, J.A. Variabilidade espacial da condutividade hidráulica e da infiltração da água no solo. Acta Scientiarum Agronomy, v.32, p.7-13, 2010

Silva, V. R.; Reichert, J. M.; Reinert, D. J. Variabilidade espacial da resistência do solo à penetração em plantio direto. Ciência Rural, v.34, p.399-406, 2004.

Statsoft, Statistica: data analysis software system. Version 7. [S. L.], 2001.

Tavares Filho, J.; Tessier, D. Effects of different management systems on porosity of oxisols in Paraná, Brasil. Revista Brasileira de Ciência do Solo, v.34, p.899-906, 2010.

Vauclin, M.; Vieira, S. R.; Vauchaud, G.; Nielsen, D. R. The use of cokriging with limited field soil observations. Soil Science Society American Journal, v.47, p.175-184, 1983.

Vieira, S. R. Geoestatística em estudos de variabilidade espacial do solo. In: Novais, R. F.; Alvares, V. H.; Schaeffer, C. E. G. R. Tópicos em ciência do solo, Viçosa: Sociedade Brasileira de Ciência do Solo, 2000. p.1-54.

Vieira, V. A. S.; Mello, C. R.; Lima, J. M. Variabilidade espacial de atributos físicos do solo em uma microbacia hidrográfica. Ciência Agrotécnica, v.31, p.1477-1485, 2007.

Warrick, A. W.; Nielsen, D. R. Spatial variability of soil physical propertiesin the field. In: Hillel, D. (ed.). Applications of soil physics. New York: Academic Press, 1980. p.319-344.

Wojciechowski, J. C.; Schumacher, M. V.; Pires, C. A. F.; Madruga, P. R. A.; Kilca, R. V.; Brun, E. J.; Silva, C. R. S.; Vaccaro, S.; Rondon Neto, R. M. Geoestatística aplicada ao estudo das características físico-químicas do solo em áreas de floresta estacional de decidual. Ciência Florestal, v.19, p.383-391, 2009.

Woodbury, A. D.; Sudicky, E. A. The geostatistical characteristics of the Borden Aquifer. Water Resources Research, v.27, p.533-546, 1991. 